\title{
ORIENTATION-PRESERVING MAPPINGS, A SEMIGROUP OF GEOMETRIC TRANSFORMATIONS, AND A CLASS OF INTEGRAL OPERATORS $\left(^{(}\right)$
}

\author{
BY \\ ANTONIO O. FARIAS( $\left.{ }^{2}\right)$
}

\begin{abstract}
A Titus transformation $T=\langle\alpha, v\rangle$ is a linear operator on the vector space of $C^{\infty}$ mappings from the circle into the plane given by $(T f)(t)=(\langle\alpha, v\rangle f)(t)$ $=f(t)+\alpha(t)$ det $\left[v, f^{\prime}(t)\right] v$, where $\alpha$ is a nonnegative, $C^{\infty}$ function on the circle $S^{1}$. Let $\tau$ denote the semigroup generated by finite compositions of Titus transformations. A Titus mapping is the image by an element of $\tau$ of a degenerate curve, $\alpha_{0} v_{0}$, where $\alpha_{0}$ is a $C^{\infty}$ function on $S^{1}$ and $v_{0}$ is fixed in the plane $R^{2}$.

A $C^{\infty}$ mapping $f: S^{1} \rightarrow R^{2}$ is called properly extendable if there is a $C^{\infty}$ mapping $F: D^{-} \rightarrow R^{2}, D$ the open unit disk and $D^{-}$its closure, such that $J_{F} \geqq 0$ on $D, J_{F}>0$ near the boundary $S^{1}$ of $D^{-}$and $\left.F\right|_{S^{1}}=f$. A $C^{\infty}$ mapping $f: S^{1} \rightarrow R^{2}$ is called normal if it is an immersion with no triple points and all its double points are transversal.

The main result of this paper can be stated: a normal mapping is extendable if and only if it is a Titus mapping.

An application is made to a class of integral operators of the convolution type, $y(t)=-\int_{0}^{2 \pi} k(s) x(t-s) d s$. It is proved that, under certain technical conditions, such an operator is topologically equivalent to Hilbert's transform of potential theory, $y(t)=\int_{0}^{2 \pi} \cot (s / 2) x(t-s) d s$, which gives the relation between the real and imaginary parts of the restriction to the boundary of a function holomorphic inside the unit disk.
\end{abstract}

0. Introduction. A map $f: S^{1} \rightarrow R^{2}$ is extendable if there is a map on the closed disk with Jacobian nonnegative throughout and positive near $S^{1}$ and which agrees with $f$ on $S^{1}$. We show that any such map can be obtained by means of a finite number of growth operations (see text for precise definitions). We also make an application to a class of convolution operators introduced by C. Loewner to show they are topologically equivalent to the Hilbert transform of potential theory.

The proof is accomplished by approximating $f$ from the inside by a map $g$ which extends to a holomorphic $G$ whose derivative has no multiple zeros, and then giving a geometric proof for such $g$.

Presented to the Society, August 19, 1970; received by the editors December 1, 1970.

AMS 1970 subject classifications. Primary 57D40, 47D05, 44A35; Secondary 30A90, 47E05, 44A15.

Key words and phrases. Normal immersions, extendable maps, holomorphic maps, Hilbert transform.

${ }^{1}$ ) The contents of this paper form a part of the author's dissertation submitted as a partial requirement for the Ph.D. degree at The University of Michigan under the direction of Professor Charles J. Titus. (Brazil).

$\left({ }^{2}\right)$ The author was supported by a scholarship from Conselho Nacional de Pesquisas 
The author wishes to express his appreciation to Professor Charles J. Titus of the University of Michigan for suggesting the problem and for his encouragement during its solution.

1. Preliminaries. If $f: A \rightarrow B$ is a function, $[f]$ denotes the image of $f$ as a subset of $B$. If $A$ is a subset of a topological space, then $A^{-}$denotes its closure.

The set of real numbers is designated by $R$.

The plane $R^{2}$ is considered both as the plane of the complex variable $z=x+i y$ and as parameterized by polar coordinates $(r, \theta)$.

We always use $D$ to denote the open unit disk in $R^{2}$ and $S^{1}$ is the naturally oriented boundary of $D . S^{1}$ will also be considered as parameterized by $\theta$ taking values in $[0,2 \pi]$; in this case, real-valued functions will be identified with functions on $R$ with period $2 \pi$.

All manifolds and maps will be smooth $\left(=C^{\infty}\right)$ unless otherwise stated. The differentiable structure of $R^{2}$ and all its submanifolds considered are the usual ones.

If $F: A \rightarrow B$ is a map between oriented manifolds, then the expression " $F$ is O.P." means that $F$ preserves orientation or, equivalently, that its Jacobian is nonnegative everywhere.

In the set $C^{\infty}\left(S^{1}, R^{2}\right)$ we consider the $C^{n}$-topology, for every $n$, induced by the norm

$$
\|f\|_{n}=\max _{i=0, \ldots, n} \max _{\theta \in S^{1}}\left|f^{(i)}(\theta)\right|
$$

where $f^{(0)}=f$ and $f^{(i)}$ is the $i$ th-derivative of $f$. The $C^{\infty}$-topology is the direct limit of all the $C^{n}$-topologies.

If $A \subset R^{2}$ and $F: A \rightarrow R^{2}$ is differentiable then $J_{F}(z)$ represents the Jacobian determinant of $F$ computed at $z \in A$.

The plane $R^{2}$ will also be considered as a vector space, with the usual structure. If $V, W \in R^{2}$ then det $[V, W]$ is the usual determinant form; thus, if $V=\left(V_{1}, V_{2}\right)$, $W=\left(W_{1}, W_{2}\right)$ then $\operatorname{det}[V, W]=V_{1} W_{2}-W_{1} V_{2}$.

\section{The topology of immersions.}

Definition 2.1. A map $f: S^{1} \rightarrow R^{2}$ is called extendable if there is a map $F: D^{-} \rightarrow R^{2}$ such that $J_{F} \geqq 0$ and $\left.F\right|_{S^{1}}=f$; in this case $F$ is called an extension of $f$. If, further, $J_{F}>0$ on $S^{1}$, then $f$ is properly extendable and $F$ is a proper extension of $f$. If $F$ is a proper extension of $f$ which is holomorphic on $D$, then $f$ is a holomorphic boundary and $F$ is a properly holomorphic map.

Proposition 2.1 [10, TheOREM 1']. Let $f$ be properly extendable. Then, there is a homeomorphism $\varphi: S^{1} \rightarrow S^{1}$ and a holomorphic boundary $g$ such that $f \cdot \varphi=g$.

LEMMA 2.1. Let $f: S^{1} \rightarrow R^{2}$ be an immersion which represents a positively oriented Jordan curve. Then, $f$ is properly extendable to an O.P.-diffeomorphism $F: D^{-} \rightarrow R^{2}$. 
Proof. Choose $\delta>0$ such that $g: S^{1} \rightarrow R^{2}$ given by $g(\theta)=f(\theta)-\delta n_{f}(\theta)$, where $n_{f}$ is the outer normal to $f$, is also an injective immersion [2, Lemma 6, p. 278]. By Francis [2, Lemma 7, p. 278], there is an orientation-preserving $C^{1}$ diffeomorphism $G: D^{-} \rightarrow R^{2}$ such that $\left.G\right|_{S^{1}}=g$ and $\left.(\partial G / \partial r)\right|_{S^{1}}=\delta n_{f}$. Define $F_{1}: D^{-} \rightarrow R^{2}$ by $F_{1}(r, \theta)=G(2 r, \theta)$ if $r \leqq \frac{1}{2}$ and $F_{1}(r, \theta)=f(\theta)-2(1-r) \delta n_{f}(\theta)$ otherwise. Then, $F_{1}$ is $C^{1}$ and $J_{F_{1}}>0$ on $D^{-},\left.F_{1}\right|_{S^{1}}=f$ and $F_{1}$ is smooth on the annulus $\frac{1}{2}<r<1$. Hence, there is a smooth $F$ with $J_{F}>0$ and which agrees with $F_{1}$ on the annulus $\frac{2}{3}<r<1$. (This can easily be obtained, for instance, from [6, Theorem 4.2 and Exercise (a)].) If $z$ is an interior point of $[F]$ we have

$$
\text { Cardinality } \begin{aligned}
F^{-1}(z) & =\sum_{y \in F^{-1}(z)} \operatorname{sgn} J_{F}(y)=\operatorname{degree}\left(\left.F\right|_{s^{1}}, z\right) \\
& =\operatorname{degree}\{\theta \rightarrow(f(\theta)-z) /|f(\theta)-z|\} \\
& =\text { tangent winding number of } g=1 . \quad \text { Q.E.D. }
\end{aligned}
$$

LEMMA 2.2. Every normal map is infinitesimally stable. (See [5], [12] for terminology.)

Proof. Let $f$ be normal and let $u$ be a vector field along $f$. We must construct vector fields $V$ on $S^{1}$ and $W$ on $R^{2}$ such that

$$
u=W \circ f+f_{*} \circ V \text {. }
$$

Clearly, it suffices to construct $W$ on a neighborhood of $[f]$.

If $f^{-1}(f(\theta))=\{\theta\}$, there is no problem, since we can take $V(\theta)$ as the unit tangent vector at $\theta$ and $W(f(\theta))=u(\theta)-f_{*} \circ V(\theta)$ and make $W$ constant along a small enough segment of the normal to $[f]$ through $f(\theta)$.

If $f\left(\theta_{1}\right)=f\left(\theta_{2}\right), \theta_{1} \neq \theta_{2}$, we need only change $V$ and $W$ in small enough neighborhoods of $\theta_{1}, \theta_{2}$ so that

$$
W\left(f\left(\theta_{1}\right)\right)=u\left(\theta_{1}\right)-f_{*} V\left(\theta_{1}\right)=u\left(\theta_{2}\right)-f_{*} V\left(\theta_{2}\right)
$$

which can be done since $f^{\prime}\left(\theta_{1}\right)$ and $f^{\prime}\left(\theta_{2}\right)$ are linearly independent, so $u\left(\theta_{1}\right)-u\left(\theta_{2}\right)$ $=C_{1} f^{\prime}\left(\theta_{1}\right)-C_{2} f^{\prime}\left(\theta_{2}\right)=f_{*}\left(C_{1}(d / d \theta)_{\theta_{1}}\right)-f_{*}\left(C_{2}(d / d \theta)_{\theta_{2}}\right)$. A smooth pasting completes the proof. Q.E.D.

The following theorem is basically a very special case of a result of Mather [5, Theorem 1, p. 267]. Mather's result, however, guarantees the existence of a neighborhood $U$ with property (1) below in the $C^{\infty}$ topology, so our theorem is a slight strengthening of Mather's result in the special case considered.

This result will be used to show that a constructed map, $g$, is $C^{\infty}$-isomorphic to a given map, $f$. Our constructions, however, only guarantee that finitely many derivatives of $g$ approximate those of $f$; thus, we cannot be sure that $f$ and $g$ are close in the $C^{\infty}$ topology and so Mather's theorem does not apply. On the other hand, $f$ and $g$, being close in the $C^{1}$ topology, are isomorphic by our theorem.

THEOREM 1 (UNIFORMIZATION THEOREM). Every normal map $f$ has a neighborhood $U=U_{f}$ in the space $C^{\infty}\left(S^{1}, R^{2}\right)$ provided with the $C^{1}$ topology, such that: 
(1) For every $g, h \in U$ there are orientation-preserving diffeomorphisms $\varphi: S^{1} \rightarrow S^{1}$, $\psi: R^{2} \rightarrow R^{2}$ such that $g \circ \varphi=\psi \circ h$.

(2) Every $g \in U$ is extendable iff there is $h \in U$ which is extendable.

Proof. Applying Lemma 2.2 to the normal field along $f$ yields a vector field, $W$, on $R^{2}$ such that $W \circ f$ and $f^{\prime}$ are linearly independent.

Letting $\Phi(X, z, t)$ denote the flow of the vector field $X$, we can find $\eta>0$ such that $\Phi(W, f(\theta), t)$ is defined for every $\theta \in S^{1}$ and $|t|<\eta$ and $f(\theta)$ is the only point in $[f] \cap\{\Phi(W, f(\theta), r):|r|<\eta\}$. The set

$$
V=\left\{z: z=\Phi(W, f(\theta), r), \theta \in S^{1},|r|<\eta\right\}
$$

is open and every one of its points is uniquely representable as $\Phi(W, f(\theta), r)$. Let

$$
V_{n}(\delta)=\{z: d(z,[f])<n \delta\}
$$

and choose $0<\delta \leqq \eta / 2$ such that $V_{2}(\delta) \subset V$.

Let $U$ be the set of all maps $g: S^{1} \rightarrow R^{2}$ such that $\|f-g\|_{1}<\delta$. This $U$ will be seen to satisfy the theorem.

In fact, if $g \in U$, a map $C_{1}: S^{1} \rightarrow R$ can be defined by the conditions

$$
\Phi\left(W, f(\theta), C_{1}(\theta)\right) \in[g], \quad\left|C_{1}(\theta)\right|<\eta,
$$

and this will induce a map $C_{2}: V_{2} \rightarrow R$ such that $C_{2}(\Phi(W, f(\theta), r))=C_{1}(\theta)$. Extend $C_{2}$ to a function $C$ on $R^{2}$ and define $W_{1}=C W, \psi(z)=\Phi\left(W_{1}, z, 1\right)$. Then, $\psi \circ f$ and $g$ are two immersions with the same image (as point sets) and orientation, hence $\psi \circ f=g \circ \varphi$, as required.

Part (2) follows from part (1) and Lemma 2.1. Q.E.D.

Proposition 2.2 (Titus, oRAL COMMUNiCATION). Every normal, extendable map is properly extendable.

Proof. Let $f$ be normal and extendable. Construct $g$ from $f$ by moving along the inward normal, say $g=f-\delta n_{f}$. By the Uniformization Theorem, if $\delta$ is small enough then $g$ is extendable, say to $G$. Use a bump function to construct a map $F$ on the disk of radius 2 which agrees with $g$ for $0 \leqq r \leqq 1$ and grows from $g=f-\delta n_{f}$ to $f$ for $1 \leqq r \leqq 2$; further, $F$ has positive Jacobian for $r>1$, provided $\delta$ is small enough. Q.E.D.

REMARK. Normality is necessary in the previous proposition, for $f(\theta)=\cos \theta$ $+i \cos ^{2} \theta \sin \theta$ is an extendable immersion which is not properly extendable.

Proposition 2.3 (TITUS, ORAL COMMUNICATION). The set of properly extendable maps is open in the $C^{1}$-topology of $C^{\infty}\left(S^{1}, R^{2}\right)$.

Proof. If $F$ is a proper extension of $f$, there is an annulus containing $S^{1}$ on which $F$ is a local diffeomorphism, and whose image is an open set $V$. The neighborhood $U$ of $f$ can be taken so that each $g \in U$ is an immersion mapping inside $V$. 
The map $\varphi=F^{-1} \circ \mathrm{g}$ is a diffeomorphism $S^{1} \rightarrow R^{2}$ which extends to a $\Phi: D^{-} \rightarrow R^{2}$ (Lemma 2.1); $G=F \circ \Phi$ is the desired proper extension of $g$. Q.E.D.

LEMMA 2.3. Let $f$ be normal, extendable and real analytic. Then, $f$ has a proper extension which is holomorphic on a neighborhood of $S^{1}$.

Proof. Since $f$ is real analytic, for each $\theta_{0}$ the function $g(\theta)=f\left(e^{i \theta}\right)$ admits a power series expansion about $\theta_{0}$; if we restrict the values of $\theta$, this will give a power series about $\exp \left(i \theta_{0}\right)$ which will converge in a disk about $\exp \left(i \theta_{0}\right)$. In the intersection of any two such disks both power series coincide with $f$ on an arc and thus they coincide in the entire intersection. In this fashion, we obtain a function $F_{1}$ holomorphic on a neighborhood of $S^{1}$ and which extends $f$.

For $r_{0}<1$ sufficiently close to 1 , the restriction of $F_{1}$ to the circle $r=r_{0}$ extends to a function, $G$, with nonnegative Jacobian. Paste $G$ and $F_{1}$ together to get an $F$ with $F=G$ for $r \leqq r_{0}$ and $F=F_{1}$ for $r \geqq r_{1}$, where $r_{0}<r_{1}<1$. Q.E.D.

\section{Titus transformations.}

Definition 3.1. A Titus transformation, $T$, is a linear operator on the vector space $C^{\infty}\left(S^{1}, R^{2}\right)$ given by

$$
(T f)(\theta)=f(\theta)+C(\theta) \operatorname{det}\left[V, f^{\prime}(\theta)\right] V,
$$

$C: S^{1} \rightarrow R$ nonnegative, $V$ fixed. We use the notation $T=\langle C, V\rangle$. The set of all finite compositions of Titus transformations is a semigroup, $\Sigma$.

The effect of a Titus transformation can be represented by an elementary operation of growth along a fixed direction, growth understood in the sense of moving to the outside of an oriented curve.

The following properties are easily verified:

(T1) Every Titus transformation is an invertible operator,

(T2) $\langle 0, V\rangle=\langle C, 0\rangle=$ identity,

(T3) $\left\langle C_{1}, V\right\rangle \circ\left\langle C_{2}, V\right\rangle=\left\langle C_{1}+C_{2}, V\right\rangle$,

(T4) $\langle C, t V\rangle=\left\langle t^{2} C, V\right\rangle$ for every real number $t$,

(T5) for $A \in \mathrm{GL}\left(R^{2}\right)$, define $A^{*} \in \mathrm{GL}\left(C^{\infty}\left(S^{1}, R^{2}\right)\right)$ by $A^{*} f=A \circ f$; then,

$$
A^{*} \circ\langle C, V\rangle \circ\left(A^{*}\right)^{-1}=\left\langle(\operatorname{det} A)^{-1} C, A V\right\rangle \text {. }
$$

In (3.1), $C$ is called the coefficient of $T$. Norton [7, Theorem 2.1, \$2.1] proved that, for the case of constant coefficients, the only relations are those expressed by T2, T3 and T4; it is clear, though, that his arguments hold for the general case as well. Thus, every $S \in \Sigma$ can be expressed uniquely as $A^{*} \circ T_{n} \circ \cdots \circ T_{1}$, where $A \in \mathrm{GL}^{+}\left(R^{2}\right)$ and each $T_{i}=\left\langle C_{i}, V_{i}\right\rangle$ is such that $\max C_{i}=1$ and $V_{i}, V_{i+1}$ are linearly independent. Hence it makes sense to talk about $S \in \Sigma$ having constant coefficients.

A "degenerate" map $S^{1} \rightarrow R^{2}$ is one whose image lies in a one-dimensional subspace. Denote by $\Delta$ the set of all such maps. 
Definition 3.2. A Titus map (or $T$-map) is a map $S^{1} \rightarrow R^{2}$ in $\Sigma(\Delta)$. The set of all $T$-maps is denoted by $\Xi$.

Thus, a $T$-map has an elementary geometric meaning: it represents a curve obtained from a "degenerate" curve by applying growth operations finitely many times.

Proposition 3.1 (Titus, oral COMmunication). Every T-map is extendable.

Proof. Let $f: S^{1} \rightarrow R^{2}$ be a $T$-map; then, it can be expressed as

$$
f=\left(A^{*} \circ T_{n} \circ \cdots \circ T_{1}\right) f_{0},
$$

where $f_{0} \in \Delta, T_{i}=\left\langle C_{i}, V_{i}\right\rangle$ and $A \in \mathrm{GL}^{+}\left(R^{2}\right)$. By induction, it suffices to prove

(a) Every $f_{0} \in \Delta$ is extendable.

(b) If $f$ is extendable and $T=\langle C, V\rangle$ is a $T$-transformation, then $T f$ is extendable.

(a) is clear, an extension of $f_{0}$ being $F(r, \theta)=\varphi(r) f_{0}(\theta)$, where $\varphi$ is a bump function going from 0 to 1 as $r$ goes from 0 to 1 .

To prove (b), let $F$ be an extension of $f$; by using the bump function $\varphi$, we can assume that $F$ is defined on the whole plane and $F(r, \theta)=f(\theta)$ for $r \geqq 1$. Define $G$ on $D^{-}$by

$$
\begin{aligned}
G(r, \theta) & =F(\varphi(2 r), \theta) & & \text { if } r \leqq \frac{1}{2}, \\
& =(\langle\varphi(2 r-1) C, V\rangle f)(\theta) & & \text { if } r \geqq \frac{1}{2} .
\end{aligned}
$$

An easy check shows that $G(1, \theta)=(T f)(\theta), J_{G}(r, \theta)=2 \varphi^{\prime}(2 r) J_{F}(r, \theta) \geqq 0$ for $r \leqq \frac{1}{2}$ and $J_{G}(r, \theta)=2 \varphi^{\prime}(2 r-1) C(\theta)\left\{\operatorname{det}\left[V, f^{\prime}(\theta)\right]\right\}^{2} / r \geqq 0$ for $r \geqq \frac{1}{2}$. Thus, $G$ is an extension of Tf. Q.E.D.

4. The classification theorem. Throughout this section, $f$ (with or without subscript) denotes a map $S^{1} \rightarrow R^{2}$.

LEMMA 4.1. If $f \in \Xi$ and $\psi$ is an O.P.-diffeomorphism of $S^{1}$, then $f \circ \psi \in \Xi$.

Proof. Direct computation.

Definition 4.1. $f_{1}$ is said to be inside $f_{2}$ iff there is a $\delta>0$ and a vector field, $V(\theta)$, along $f_{1}$ such that

(1) $\left\{f_{1}(\theta)+t V(\theta):|t|<\delta\right\}$ has exactly one point in common with either $\left[f_{1}\right]$ or $\left[f_{2}\right]$,

(2) $\operatorname{det}\left[V, f_{i}^{\prime}\right]>0, i=1,2$.

Using the direction $V(\theta)$ to grow locally from $f_{1}$ to $f_{2}$ in a neighborhood of $f_{1}(\theta)$, and the compactness of $S^{1}$, we get the following.

LEMMA 4.2. If $f_{1}$ is inside $f_{2}$, there is $T \in \Sigma$ such that $f_{2}=T f_{1}$.

If $F: U \rightarrow R^{2}, U$ open in $R^{2}$, is a map with $J_{F}>0$ on some circle $r=r_{0}$, a local argument and compactness will show the existence of an $\varepsilon>0$ such that whenever $r_{0}-\varepsilon \leqq r_{1}<r_{2} \leqq r_{0}+\varepsilon$ then $\left.F\right|_{r=r_{1}}$ is inside $\left.F\right|_{r=r_{2}}$. Again by compactness, Lemma 4.2 yields 
Proposition 4.1. Let $V$ be an open set containing the annulus $0<a \leqq r \leqq b$, and let $F \in C^{\infty}\left(V, R^{2}\right)$ with $J_{F}>0$ on $V$. Then, there is $T \in \Sigma$ such that $\left.F\right|_{r=b}=T\left(\left.F\right|_{r=a}\right)$.

LEMMA 4.3. Let $F$ be a conformal diffeomorphism of the disk of radius 2 into $R^{2}$ such that $F\left(D^{-}\right)$is a convex set whose boundary has positive curvature everywhere. Then, the restriction of $F$ to $S^{1}$ is a T-mapping.

Proof. The lemma is proved by a direct geometric construction; we project the image of $S^{1}$ onto a diameter of the convex set $F\left(D^{-}\right)$along a direction perpendicular to that diameter. Q.E.D.

The Implicit Function Theorem together with Proposition 4.1 and Lemma 4.3 yield a proof of

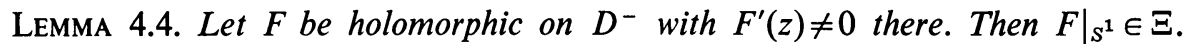

Proposition 4.2. Let $F$ be holomorphic on $D^{-}$and assume $F^{\prime}$ has only simple zeros there. Then, $\left.F\right|_{S^{1}} \in \Xi$.

Proof. Let us assume first that $F^{\prime}$ has exactly one zero, say, at $z_{0} \in D$.

Since $F^{\prime \prime}\left(z_{0}\right) \neq 0, F$ behaves locally as $G(z)=z^{2}$. Thus, we can choose $a<\left|z_{0}\right|<b$ such that the images of the circles $|z|=a,|z|=z_{0}$ and $|z|=b$ are (locally) as in Figure 1. Let $f_{1}, f_{2}, f_{3}$ be the restrictions of $F$ to these circles, respectively. It is clear how to go from $f_{1}$ to $f_{3}$ : first, move $f_{1}$ so that the new map, say $g_{1}$, goes through $F\left(z_{0}\right)$; then create $g_{2}$ inside $f_{2}$ (in the geometric sense) with a cusp (simple zero of $f_{2}^{\prime}$ ) at $z_{0}$; a third growth step will create an immersion $g_{3}$ inside $f_{3}$ (in the sense of

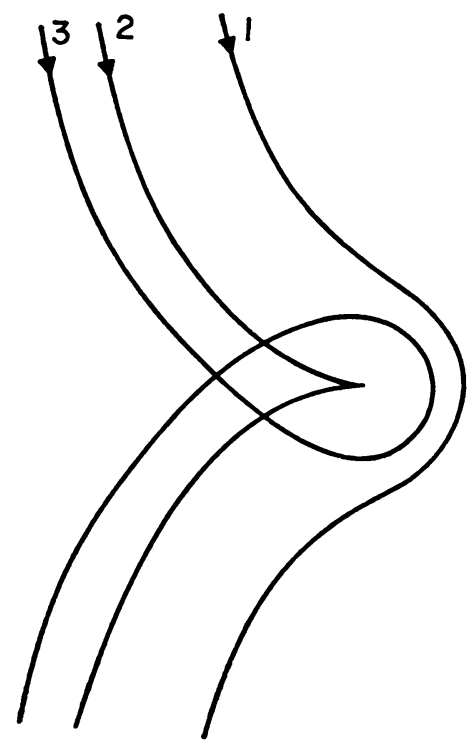

FIGURE 1. Image by $F$ of circles near a zero of $F^{\prime}$ 
Definition 4.1), and use Lemma 4.2 to see that $f_{3}=T f_{1}$ for some $T \in \Sigma$. The result in this case follows now from Lemma 4.4 and Proposition 4.1. Since $F^{\prime}$ has only finitely many zeros, an iteration of the argument above yields the result in the general case. Q.E.D.

LEMMA 4.5. Every holomorphic boundary is a T-map.

Proof. Let $F$ be a proper holomorphic extension of $f$. Approximate $F$ by a proper holomorphic map $G$ satisfying: (i) $G^{\prime}$ has only simple zeros; and (ii) the restriction of $G$ to $S^{1}$ is inside $f$ ( $G$ can be a polynomial approximation to $F$, for instance). Use Proposition 4.2 and Lemma 4.2. Q.E.D.

LEMmA 4.6. Every normal, real analytic, extendable immersion is a T-map.

Proof. Let $f$ be a normal, real analytic, extendable immersion. By Lemma 2.3, there is a disk $U \supset D^{-}$, an annulus $U \supset V \supset S^{1}$ and a map $F: U \rightarrow R^{2}$ holomorphic in $V$ and with $J_{F}>0$ on $V$. By [10, Theorem $1^{\prime}$ and proof of Lemma 4], there is an interior map $I: U \rightarrow R^{2}$ such that $\left.I\right|_{V}=\left.F\right|_{V}$.

By [13, p. 103] there is a homeomorphism $H_{1}: U \rightarrow U$ and a holomorphic map $F_{1}: U \rightarrow R^{2}$ such that $I=F_{1} \circ H_{1}$.

By [9, Theorem 2] there is a homeomorphism $H_{2}: D^{-} \rightarrow D^{-}$and a properly holomorphic map $F_{2}: D^{-} \rightarrow R^{2}$ such that $\left.I\right|_{D^{-}}=F_{2} \circ H_{2}$. From Stoilow's proof, we can take $\left.F_{1}\right|_{D}=F_{2},\left.H_{1}\right|_{D}=H_{2}$.

Since $\left.F_{1}\right|_{V}=\left.I \circ H_{1}^{-1}\right|_{V}=\left.F \circ H_{1}^{-1}\right|_{V},\left.F_{1}\right|_{V}$ is a local homeomorphism, thus a local diffeomorphism [13, Chapter VII, Corollary 1.21]. Since $H_{1}=F_{1}^{-1} \circ F$ locally, $\left.H_{1}\right|_{V}$ is a diffeomorphism. Since by continuity $f=\left(\left.F_{1}\right|_{s^{1}}\right) \circ\left(\left.H_{1}\right|_{s^{1}}\right), f \in \Xi$, by Lemmas 4.5 and 4.1. Q.E.D.

THEOREM 2. Every properly extendable map is a T-map.

Proof. Let $f$ be properly extendable.

Since the normal, real analytic immersions are open and dense ([12], [2], [8]), it is easy to see that $f$ is the limit of a sequence of such maps, each of which is inside $f$. The theorem then follows from Lemmas 4.6 and 4.2. Q.E.D.

Theorem 3 (Classification Theorem). A normal immersion is extendable iff it is a T-map.

Proof. Theorem 2, Proposition 3.1 and Proposition 2.2. Q.E.D.

5. An application to integral operators. It is a simple fact (which can be obtained, for instance, from [4, Lemma 2]) that an extendable map represents a curve of nonnegative circulation (i.e., nonnegative winding number about any point of the plane not on its graph). The converse, however, is not true; the map in Figure 2 (under a suitable parametrization) is normal and has nonnegative circulation, but it is not extendable $\left({ }^{3}\right)$.

$\left({ }^{3}\right)$ Its tangent winding number is 0 , while for any normal extendable map the tangent winding number is at least 1 (see [9]). The example is due to Titus. 
Thus, it is natural to ask when a map of nonnegative circulation is extendable. Our methods can give a positive answer under fairly general assumptions. Clearly, it is desirable that these assumptions be reasonably simple and include at least the holomorphic boundaries, the nicest maps which are both extendable and of nonnegative circulation.

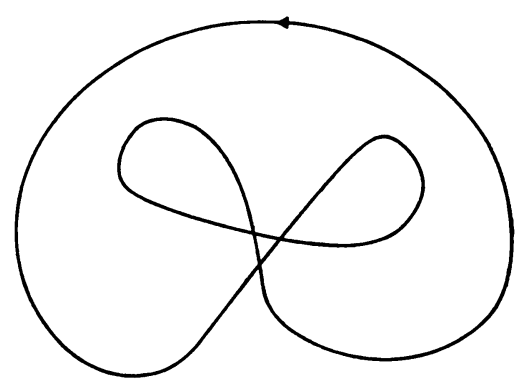

FIGURE 2. Example of a curve with nonnegative circulation which is not extendable

Consider the operator on the set of continuous functions with period $2 \pi$ given by

$$
y(t)=-\int_{0}^{2 \pi} k(s) x(t-s) d s,
$$

where $k$ is integrable in [0,2 $]$ ]. Loewner [4] proved that a necessary and sufficient condition for the plane curve $x=x(t), y=y(t)$ given by (5.1) to have nonnegative circulation for every such $x$ is that (after a possible change on a set of measure zero) $k$ be real analytic on $(0,2 \pi)$ with derivative given by

$$
k^{\prime}(s)=\int_{-\infty}^{\infty} e^{-s r} d \mu(r), \quad 0<s<2 \pi,
$$

the integral taken in the sense of Stieltjes-Lebesgue relative to a nondecreasing function $\mu$.

If $F\left(e^{i \theta}\right)=x(\theta)+i y(\theta)$ is a holomorphic boundary, then by the Hilbert transform

$$
y(\theta)=\mathrm{P} . \mathrm{V} \cdot \int_{0}^{2 \pi} \cot (s / 2) x(t-s) d s,
$$

provided $y(0)=0$. Here, the letters P.V. mean the integral must be taken in the sense of Cauchy's principal value (see [3]).

We note that (5.3) is closely related to $(5.1)$, since

$$
\frac{d}{d s}(-\cot s)=\csc ^{2} s=\int_{-\infty}^{\infty} \frac{t e^{-s t}}{1-e^{-\pi t}} d t
$$

$[14$, p. 260] and

$$
\mu^{\prime}(t)=t /\left(1-e^{-\pi t}\right) \geqq 0 .
$$

The only difference is that $k(s)=\cot (s / 2)$ is not integrable, so (5.1) becomes a singular integral. 
From what has been said, it is natural to consider maps $f: S^{1} \rightarrow R^{2}$ given by $f(\theta)=x(\theta) V_{1}+y(\theta) V_{2}$ where $V_{1}, V_{2}$ is a positively oriented base and

$$
y(\theta)=-\mathrm{P} . \mathrm{V} \cdot \int_{0}^{2 \pi} k(\tau) x(\theta-\tau) d \tau,
$$

where $k$ satisfies the following conditions:

(i) $k^{\prime}$ is given by $(5.2)$;

(ii) $k(s)=h(s) / s^{\alpha}(2 \pi-s)^{\alpha}$ for some $0 \leqq \alpha \leqq 1$ and some function $h$ Lebesgue integrable in $[0,2 \pi]$;

(iii) P.V. $\int_{0}^{2 \pi} k(s) d s$ exists and is finite;

(iv) If $\alpha>0, \int_{0}^{2 \pi} k(s) d s$ is not defined.

By [1], [4] all such maps have nonnegative circulation; they will be called $B L$ maps. From the paragraph above, every holomorphic boundary is a BL-map; we will show that, up to homeomorphisms, the converse is true.

From the work of Benson, Loewner and Norton ([1], [4], [7]) we can conclude:

Proposition 5.1. The set of T-maps with constant coefficients is dense in the set of BL-maps under the $C^{1}$-topology.

Proposition 5.2. Every normal BL-map is properly extendable.

Proof. Proposition 5.1 and the Uniformization Theorem.

From this and Proposition 2.1 we get:

THEOREM 4. Every normal, BL-map is, after a topological change of parameter, a holomorphic boundary.

This means that, given a normal BL-map $f$ there is an O.P.-homeomorphism $\varphi: S^{1} \rightarrow S^{1}$ and a properly holomorphic map $F: D^{-} \rightarrow R^{2}$ such that $\left.F\right|_{S^{1}}=f \circ \varphi$.

\section{REFERENCES}

1. D. C. Benson, Extensions of a theorem of Loewner on integral operations, Pacific J. Math. 9 (1959), 365-377. MR 21 \#7406.

2. G. K. Francis, The folded ribbon theorem. A contribution to the study of immersed circles, Trans. Amer. Math. Soc. 141 (1969), 271-303. MR 39 \#4863.

3. O. D. Kellogg, Unstetigkeit in den Integralgleichung, Math. Ann. 58 (1904), 441-456.

4. C. Loewner, A topological characterization of a class of integral operators, Ann. of Math. (2) 49 (1948), 316-332. MR 9, 502.

5. J. N. Mather, Stability of $C^{\infty}$ mappings. II. Infinitesimal stability implies stability, Ann. of Math. (2) 89 (1969), 254-291. MR 41 \#4582.

6. J. R. Munkres, Elementary differential topology, rev. ed., Ann. of Math. Studies, no. 54, Princeton Univ. Press, Princeton, N. J., 1966. MR 33 \#6637.

7. V. T. Norton, Jr., On polynomial and differential transvections of the plane, Dissertation, University of Michigan, Ann Arbor, Mich., 1970.

8. C. J. Titus, A theory of normal curves and some applications, Pacific J. Math. 10 (1960), 1083-1096. MR 22 \#5014.

9. - The combinatorial topology of analytic functions on the boundary of a disk, Acta Math. 106 (1961), 45-64. MR 29 \#3652. 
10. C. J. Titus, Characterizations of the restriction of a holomorphic function to the boundary of a disk, J. Analyse Math. 18 (1967), 351-358. MR 35 \#3072.

11. C. J. Titus and G. S. Young, An extension theorem for a class of differential operators, Michigan Math. J. 6 (1959), 195-204. MR 22 \#231.

12. H. Whitney, On regular closed curves in the plane, Compositio Math. 4 (1937), 276-284.

13. G. T. Whyburn, Topological analysis, 2nd rev. ed., Princeton Math. Series, no. 23, Princeton Univ. Press, Princeton, N. J., 1964. MR 29 \#2758.

14. D. V. Widder, The Laplace transform, Princeton Math. Series, vol. 6, Princeton Univ. Press, Princeton, N. J., 1941. MR 3, 232.

Department of Mathematics, Eastern Michigan University, Ypsilanti, Michigan 48197 